\title{
Egr-1 mediates the suppressive effect of IL-1 on PPARy expression in human OA chondrocytes
}

\author{
Salwa Sarah Nebbaki ${ }^{*}$, Fatima Ezzahra El Mansouri ${ }^{1}$, Nadia Zayed ${ }^{1}$, Mohamed Benderdour $^{2}$, \\ Johanne Martel-Pelletier ${ }^{1}$, Jean-Pierre Pelletier ${ }^{1}$, Hassan Fahmi ${ }^{1}$ \\ From 7th European Workshop on Immune-Mediated Inflammatory Diseases \\ Noordwijk aan Zee, the Netherlands. 28-30 November 2012
}

\section{Background}

Peroxisome proliferator-activated receptor gamma (PPAR $\gamma$ ) is a ligand activated transcription factor and member the nuclear hormone receptor superfamily. Several lines of evidence indicate that PPAR $\gamma$ have protective effects in osteoarthritis (OA). Indeed, PPAR $\gamma$ has been shown to down-regulate several inflammatory and catabolic responses in articular cartilage and chondrocytes and to be protective in animal models of OA.

\section{Aim}

We have previously shown that IL-1 down-regulated PPAR $\gamma$ expression in OA chondrocytes. In the present study we will investigate the mechanisms underlying this effect of IL-1.

\section{Methods}

Chondrocytes were stimulated with IL-1, and the level of PPAR $\gamma$ and Egr-1 protein and mRNA were evaluated using Western blotting and real-time reverse-transcription polymerase chain reaction, respectively. The PPAR $\gamma$ promoter activity was analyzed in transient transfection experiments. Egr-1 recruitment to the PPAR $\gamma$ promoter was evaluated using chromatin immunoprecipitation (ChIP) assays. Small interfering RNA (siRNA) approaches were used to silence Egr-1 expression.

\section{Results}

We demonstrated that the suppressive effect of IL-1 on PPAR $\gamma$ expression requires de novo protein synthesis and was concomitant with the induction of the transcription factor Egr-1. ChIP analyses revealed that IL-1 induced Egr-1 recruitment at the PPAR $\gamma$ promoter. IL-1 inhibited the activity of PPAR $\gamma$ promoter and overexpression of Egr-1 potentiated the inhibitory effect of IL-1, suggesting that Egr-1 may mediate the suppressive effect of IL-1. Finally, Egr-1 silencing with small interfering RNA blocked IL-1-mediated down-regulation of PPAR $\gamma$ expression.

\section{Conclusion}

These results indicate that Egr-1 contributes to IL-1mediated down-regulation of PPAR $\gamma$ expression in OA chondrocytes and suggest that this pathway could be a potential target for pharmacologic intervention in the treatment of $\mathrm{OA}$ and possibly other arthritic diseases.

\section{Author details}

'Osteoarthritis Research Unit, University of Montreal Hospital Research Center (CRCHUM), Notre-Dame Hospital, Montreal, Quebec, Canada. ${ }^{2}$ Centre de recherche Hôpital Sacré-Coeur de Montréal, Montreal, Quebec, Canada.

Published: 28 November 2012

doi:10.1186/1479-5876-10-S3-P39

Cite this article as: Nebbaki et al:: Egr-1 mediates the suppressive effect of IL-1 on PPAR $\gamma$ expression in human OA chondrocytes. Journal of Translational Medicine 2012 10(Suppl 3):P39. 